\title{
Photoacoustic computed tomography of human extremities
}

\author{
Parker Wray \\ Li Lin \\ Peng $\mathrm{Hu}$ \\ Lihong V. Wang
}




\title{
Photoacoustic computed tomography of human extremities
}

\author{
Parker Wray, ${ }^{a, \dagger}$ Li Lin, ${ }^{b, \dagger}$ Peng Hu, ${ }^{c}$ and Lihong V. Wang ${ }^{a, b, *}$ \\ ${ }^{a}$ California Institute of Technology, Caltech Optical Imaging Laboratory, Department of Electrical Engineering, Pasadena, California, United States \\ ${ }^{\mathrm{b}}$ California Institute of Technology, Caltech Optical Imaging Laboratory, Andrew and Peggy Cheng Department of Medical Engineering, \\ Pasadena, California, United States \\ ${ }^{c}$ Washington University in St. Louis, Department of Biomedical Engineering, St. Louis, Missouri, United States
}

\begin{abstract}
We present a method of imaging angiographic structures in human extremities, including hands, arms, legs, and feet, using a newly developed photoacoustic computed tomography (PACT) system. The system features deep penetration (1.8 cm in muscular tissues) with high spatial and temporal resolutions. A volumetric image is acquired within 5 to $15 \mathrm{~s}$ while each cross sectional image is acquired within $100 \mu \mathrm{s}$. Therefore, we see no blurring from motion in the imaging plane. Longitudinal and latitudinal cross-sectional images of a healthy volunteer clearly show the vascular network of each appendage and highlight the system's ability to image major and minor vasculatures, without the use of an external contrast or ionizing radiation. We also track heartbeatinduced arterial movement at a two-dimensional frame rate of $10 \mathrm{~Hz}$. This work substantiates the idea that PACT could be used as a noninvasive method for imaging human vasculatures. $\odot$ The Authors. Published by SPIE under a Creative Commons Attribution 4.0 Unported License. Distribution or reproduction of this work in whole or in part requires full attribution of the original publication, including its DOI. [DOI: 10.1117/1.JBO.24.2.026003]
\end{abstract}

Keywords: photoacoustic computed tomography; angiography; appendicular imaging; dual-sided illumination; panoramic photoacoustic detection

Paper 180458SSR received Jul. 24, 2018; accepted for publication Jan. 29, 2019; published online Feb. 19, 2019.

\section{Introduction}

Vascular disease is the number one killer of people in the United States, accounting for one in every three deaths. ${ }^{1}$ Within this category, vascular disease present in appendicular regions is among the most common, and it doubles the likelihood of a vascular related death. ${ }^{1,2}$ The ideal modality for imaging vascular health would provide images with strong vascular contrast, high spatiotemporal resolutions, a sufficient penetration depth, and complete noninvasiveness. ${ }^{1-11}$ In this respect, photoacoustic computed tomography (PACT) holds promise as a method of appendicular vascular imaging.

Current clinical methods of angiographic imaging include digital subtraction angiography (DSA), computed tomography angiography (CTA), magnetic resonance angiography (MRA), ultrasonography, and optical coherence tomography angiography (OCTA). ${ }^{4-17}$ Of these modalities, catheter DSA is considered the reference standard. ${ }^{6-8}$ It provides strong vascular contrast, even in a dense soft tissue or bony environment, and can acquire images over a large field of view. ${ }^{8}$ Inherent drawbacks to this method include the use of ionizing radiation and an exogenous contrast agent (often an injected iodine-bound compound). ${ }^{5-7}$ These factors can create severe complications, particularly for patients who are pregnant, young, allergic to contrast agents, impaired in renal functions, or susceptible to other contrast-induced side effects. ${ }^{5}$ With the advances in multidetector row computed tomography (CT) and MRI technologies, contrast-enhanced MRA and CTA have largely replaced DSA for diagnostic purposes. These modalities offer significantly reduced cost and increased patient comfort, compared

\footnotetext{
*Address all correspondence to Lihong V. Wang, E-mail: LVW@caltech.edu
}

tThese authors contributed equally to this work. to DSA, as well as the ability of three-dimensional (3-D) imaging, although the same fundamental drawbacks are still present. ${ }^{6,11}$ CTA requires the use of ionizing radiation (approximately four times less than DSA), and contrast-based subtraction techniques are still needed for dense soft tissue or bony areas. ${ }^{6,11}$ MRA can be devoid of ionizing radiation and capable of endogenously imaging blood vessels. However, endogenous MRA images suffer from a low contrast-to-noise ratio, therefore requiring long scan times with added cost. ${ }^{5,6}$ Ultimately the spatial resolution of these images is limited due to blurring of nonstationary vessels and other motion artifacts. ${ }^{5,6}$ If spatial resolution is insufficient, vascular abnormalities such as stenoses may be underestimated. ${ }^{6}$ Because of this, the standard for MRA is still to use a contrast agent, such as gadolinium-based compounds, at the risk of similar complications present in DSA (e.g., pregnancy, allergies, and renal insufficiency). ${ }^{12,13}$ Duplex ultrasound is often recommended as the first modality to investigate peripheral vascular structures because it is cost effective and noninvasive. ${ }^{4,8,10}$ This method uses traditional ultrasonography to image blood vessels in conjunction with Doppler ultrasound to measure aspects of blood flow, such as blood flow speed. However, ultrasonography has low sensitivity due to a lack of strong vascular contrast, suffers from speckle artifacts, and its reliability is subject to the operator's experience. ${ }^{8,14}$ OCTA is a noninvasive optical imaging technique that has seen great clinical popularity for imaging microvasculature, particularly in the eye, and shows great promise for dermatology applications. ${ }^{15-17}$ Though this technique has a shallow penetration depth and is currently not suitable for noninvasive imaging of vasculature beyond one millimeter depth. ${ }^{16,17}$ PACT is a promising modality that avoids many of these limitations.

PACT-ultrasonically imaging optical contrast via the photoacoustic (PA) effect-provides the rich contrast of optical imaging well beyond the $\sim 1-\mathrm{mm}$ optical diffusion limit, with 
ultrasonic resolution and no speckle artifact. ${ }^{18-21}$ As a highly scalable technology, PACT can perform structural, functional, and molecular imaging. ${ }^{18-21}$ When a short-pulsed laser irradiates biological tissues, wideband ultrasonic waves (referred to as PA waves) are induced by transient thermoelastic expansion. The PA waves are then measured by ultrasound transducers and reconstructed to produce a map of the specific absorption $\left(\mathrm{J} / \mathrm{m}^{3}\right)$ in the tissue. ${ }^{21}$ As a principal endogenous optical absorber in the near-infrared (NIR) region, hemoglobin provides a strong endogenous contrast for imaging blood vessels in human extremities. $^{22}$

We present the results of imaging the angiographic structure of the hands, arms, legs, and feet of a healthy volunteer, using a recently developed PACT system. Longitudinal and latitudinal cross-sectional images clearly show the vascular network of each appendage and highlight the system's ability to image both major and minor vessels. Images are captured within 5 to $15 \mathrm{~s}$, without the need for an experienced operator. The work shows that PACT could be a noninvasive method for providing strong contrast angiographic images of human extremities with high spatiotemporal resolutions, suggesting that it could provide a useful modality for detecting vascular disease and monitoring vascular health.

\section{Methods}

The PACT system was comprised of an illumination laser, an ultrasonic transducer array, signal amplification/acquisition modules, and a linear scanning stage (Fig. 1). PA signals were generated using $1064 \mathrm{~nm}$ light (PRO-350-10, Quanta-Ray, $10-\mathrm{Hz}$ pulse repetition rate, 8 to 12 -ns pulse width) and detected using a full-ring ultrasonic transducer array with 512 elements. Four sets of 128-channel data acquisition systems provided simultaneous one-to-one mapped associations with the 512element transducer array. Therefore, we acquired PA signals from a cross-section within $100 \mu \mathrm{s}$, approximately the acoustic transit time across the field of view after each laser pulse excitation, without multiplexing. The ultrasonic transducer elements had a central frequency of $2.25 \mathrm{MHz}$ and a one-way bandwidth of more than $95 \%$, providing an experimentally quantified in-plane resolution of $255 \mu \mathrm{m} .^{23}$ The height of each transducer element yielded a moderate divergence angle in the elevational direction ( $\sim 9.0 \mathrm{deg}$ full width at half maximum), yielding a flared diffraction pattern. ${ }^{23}$ This pattern enabled both twodimensional (2-D) imaging per laser pulse and 3-D imaging by scanning in the elevational direction. From multislice PA signals acquired through elevational scanning, a 3-D image can be formed by slicewise 2-D universal backprojection (UBP) and stacking or cylindrical 3-D UBP. The elevational resolution in the reconstructed image is $16.1 \mathrm{~mm}$ for 2-D reconstruction and stacking and $5.6 \mathrm{~mm}$ for $3-\mathrm{D}$ reconstruction. ${ }^{23}$ Full characterization measurements for this system can be found in Ref. 23 . To synchronize the system, the laser's external trigger was used to initiate both the data acquisition systems and the linear scanner.

For each appendage, dual-side illumination was used to take latitudinal cross-sectional images [Fig. 1(a)] and longitudinal cross-sectional images [Fig. 1(b)]. A switchable mirror was used to alternate between the two illumination schemes. To split the laser beam, the linearly polarized output from the laser was equalized between $s$ - and $p$-polarizations, using a half-wave plate (WPH10M-1064, Thorlabs, Inc.), and then divided using a high-power polarizing beam splitter (PBS25-1064-HP, Thorlabs, Inc.). A center incidence angle of $55 \mathrm{deg}$ was chosen to couple light into the water to ensure that (a) the illumination covered the entire $z$-axis scanning range $(7 \mathrm{~cm})$, (b) the transducer array did not impede the illumination when scanning, and (c) the proximity to Brewster's angle provided a high light coupling efficiency for $p$-polarization. To maximize and equalize the fluence received from each beam, the $s$-polarized path was passed through another half-wave plate (WPH10M-1064, Thorlabs, Inc.), ensuring both paths were $p$-polarized before hitting the water surface. This optimization provided an average air-water coupling efficiency of $98 \%$ over a $55 \pm 20$ - deg angular range. Before hitting the water surface, each beam was broadened using a diverging lens (LC1315-B, Thorlabs, Inc.) and an engineered diffuser (EDC-10-A-2s, RPC Photonics), which preserved polarization. For all scans, the laser fluence at the tissue surface was between $1 / 16$ th and $1 / 8$ th of the American (a)

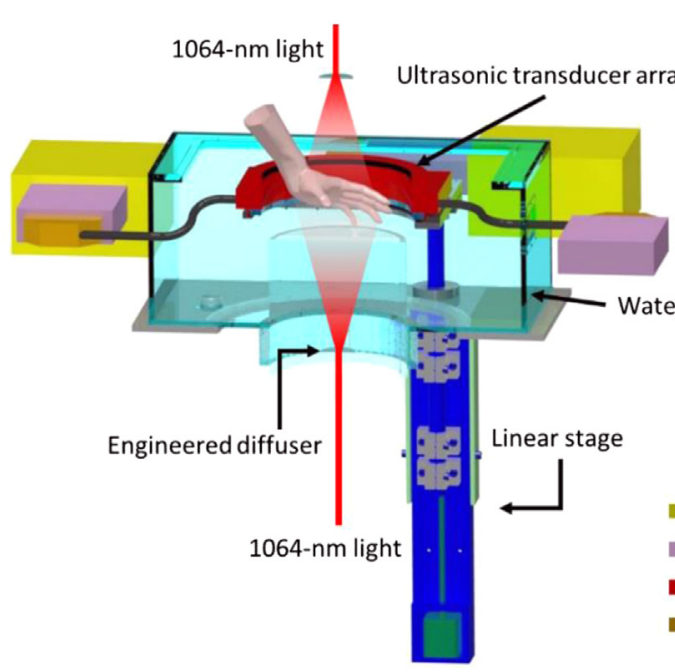

(b)

\section{Longitudinal Mode}

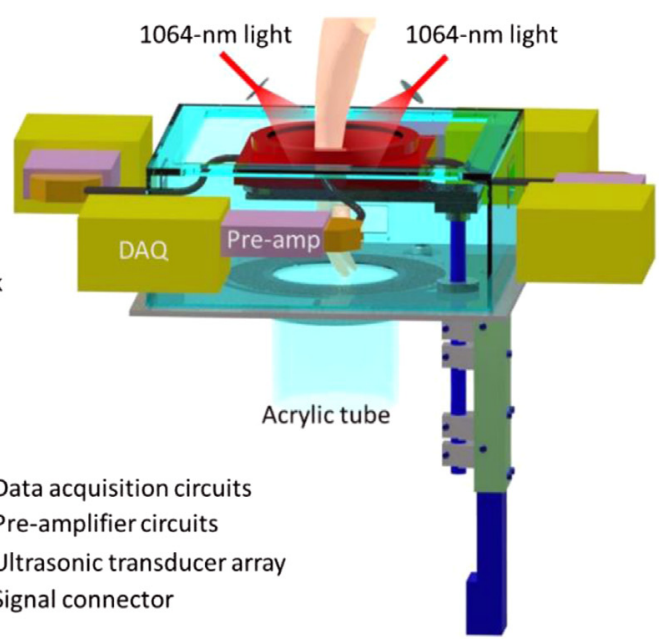

Fig. 1 Representations of the PACT system under different illumination schemes. Perspective cut-away views of the PACT system using (a) latitudinal mode illumination and (b) longitudinal mode illumination. 
National Standards Institute (ANSI) safety limit for laser exposure $\left(100 \mathrm{~mJ} / \mathrm{cm}^{2}\right.$ at $1064 \mathrm{~nm}$ at a $10-\mathrm{Hz}$ pulse repetition rate). ${ }^{24}$

Images were reconstructed using the UBP method with halftime truncation. ${ }^{25,26}$ For 2-D reconstruction, PA signals generated from a laser pulse were backprojected to the imaging plane using the data from all 512 transducers in the ring array. For 3-D reconstruction, each imaging plane was reconstructed using angle-weighted data from every ultrasonic transducer element at all scanning steps. The finite elevational divergence to the transducer array was approximated by taking an integer power of the angle weight associated with a uniform far field angular profile (i.e., the profile of a theoretical perfectly diverging transducer). With 512 transducer elements, the full-ring transducer array can image objects without streaking artifacts, within a field-of-view (FOV) of $\sim 39 \mathrm{~mm}$ in diameter. The relationship is governed by the equation $2 \pi D=N \lambda_{c}$, where $D$ is the FOV diameter, $N$ is the number of elements, and $\lambda_{c}$ is the wavelength corresponding to the high cut-off frequency of the transducer. ${ }^{27,28}$ To suppress backprojection-induced streaking artifacts outside of this FOV, we interpolated the raw data in the channel dimension before doing UBP. ${ }^{29}$ After reconstruction, total variation and Wiener filtering were used to reduce noise while preserving sharp features. ${ }^{30}$ To highlight blood vessels, we used the process detailed in Ref. 28, where Hessian-based Frangi vesselness filtering was used with scales of 5 to 10 representative values within the range of the smallest and largest vessels in each image. ${ }^{31-33}$ All quantitative analysis, including analysis of vessel sizes and maximum penetration depth, was based on unfiltered images. Therefore, filtering effects such as blurring or enhancement of contrast did not alter the quantitative results.

All human imaging followed protocols approved by the Institutional Review Board (IRB) and Protocol Review of California Institute of Technology. One healthy volunteer was imaged multiple times in this study.

\section{Results}

Figure 2 shows a 3-D reconstructed maximum amplitude projection (MAP) and corresponding color-encoded depth-resolved image of a volunteer's right hand using latitudinal mode illumination. The projection is along the $z$-axis. The shallow region is the palmar side and the deeper region is the dorsal side of the hand. Vessels can be visualized starting from the dorsal side of the wrist and ending at the palmar side of the fingers. The vessel network can be clearly tracked across the entire hand with aberration occurring when trying to look through the fingernails.

To further demonstrate PACT's ability to image vasculature deep into biological tissue, we scanned cross-sections of the volunteer's right forearm and hand (Fig. 3), using longitudinal mode illumination. Throughout the scan, we imaged vasculature up to $1.8 \mathrm{~cm}$ deep in all directions (Video 1). We also found that by only using time data corresponding to the propagation distance from the transducer surface to the center of the imaging plane (i.e., half-time reconstruction ${ }^{25}$ ), the effect of acoustic inhomogeneity from the bone-tissue interface could be reduced. This observation highlights deep vasculature imaging without the need for an external contrast agent as a potential application of PACT.

The maximum imaging depth in muscular tissue from a single PACT image (i.e., a single laser pulse) was experimentally determined to be $1.8 \mathrm{~cm}$, by imaging the volunteer's right calf (Fig. 4). We study muscular imaging depth in the calf because the gastrocnemius is a large section of isolated muscle tissue. The fluence used was the maximum fluence for this study (1/8th of the ANSI safety limit) and corresponded to a beam size just covering the circumference of the calf. To estimate the optical penetration, a distribution of the specific absorption in bulk muscle tissue was approximated by extracting the background PACT image [Fig. 4(a)]. The image was made using morphological background detection, ${ }^{34}$ which is a process that removes blood vessels and other sharp features by top-hat transforms and Gaussian filtering $(\sigma=20$ for a $2033 \times 2033$ pixel image with a resolution of $30 \mu \mathrm{m}$ per pixel). A benefit of this method is that it can be used after PACT reconstruction and on objects with a complex geometry and/or under complex illumination conditions, such as double-sided illumination. To assess the validity of this method, we generate statistics of the amplitude decay from four regions in Fig. 4(a). We then compare the average decay from these regions to Monte Carlo simulated specific absorption in muscular tissue [Fig. 4(c)], without and with the background removal process used in Fig. 4(a) applied. The latter comparison is performed to quantify the broadening effects of the background removal process. We found that background removal caused negligible broadening of the absorption profile inside the simulated muscular tissue and that the average



Fig. 2 Photoacoustic images of the volunteer's right hand from 3-D reconstruction. (a) MAP of the PA signals along the $z$-axis. (b) Color encoded depth image of the hand. 

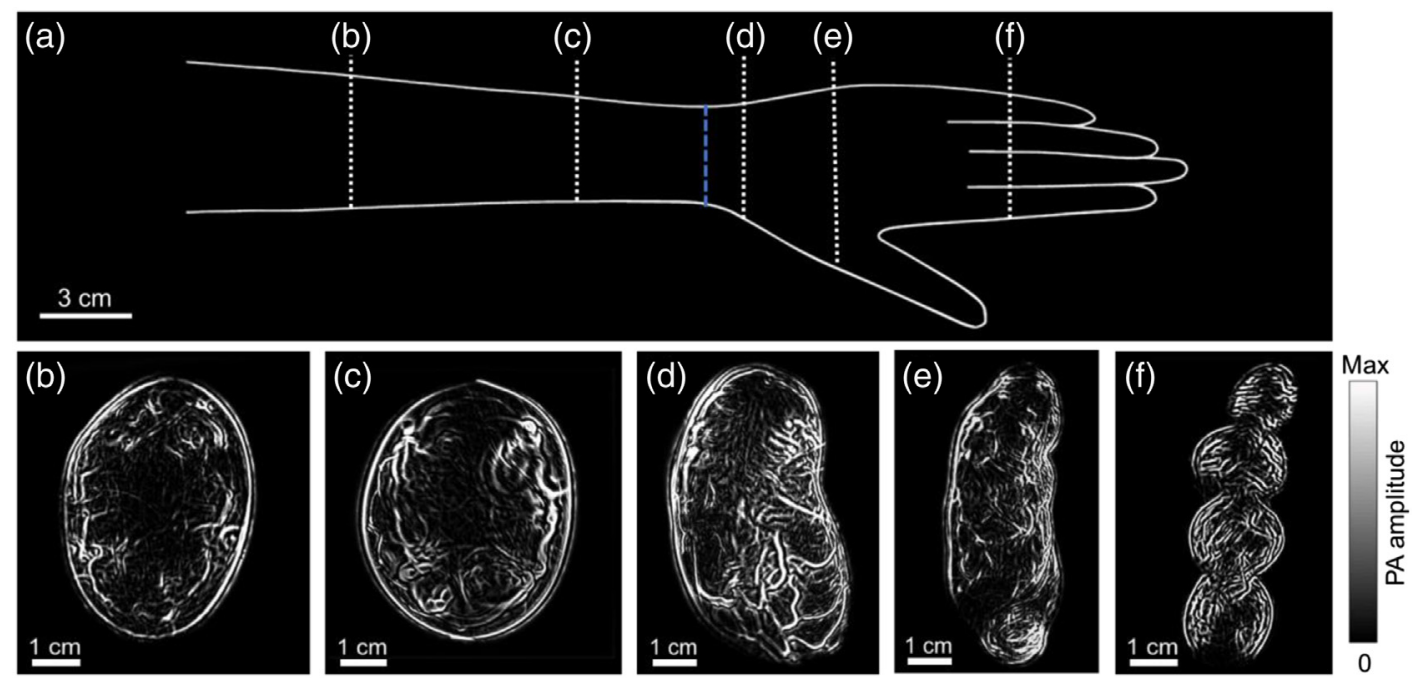

Fig. 3 Lateral cross-sectional PA images acquired at various locations along the volunteer's right arm (Video 1). (a) Schematic of the arm. White dotted lines detail where each cross-sectional image was taken for images (b)-(f), respectively. The blue dashed line marks the wrist, where the carpal bones meet the radius and ulna bones. Lateral cross-sectional PA images of (b) the upper forearm, $10 \mathrm{~cm}$ above the wrist, (c) the lower forearm, $3 \mathrm{~cm}$ above the wrist, (d) the palm, $1 \mathrm{~cm}$ below the wrist, (e) the mid-palm, $3 \mathrm{~cm}$ below the wrist, and (f) the fingers (without the thumb), approximately in the middle of the proximal phalanx (Video 1, MPEG, 2 MB [URL: https://doi.org/10.1117/1.JBO.24.2.026003.1]).

(a)

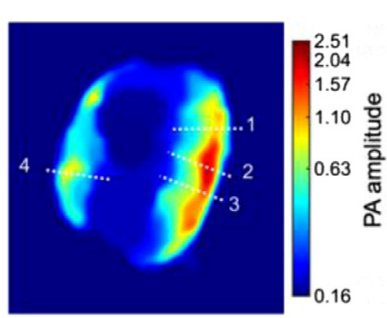

(d)

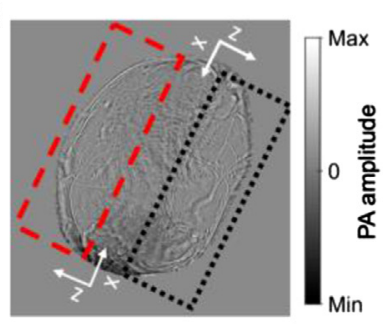

(b)

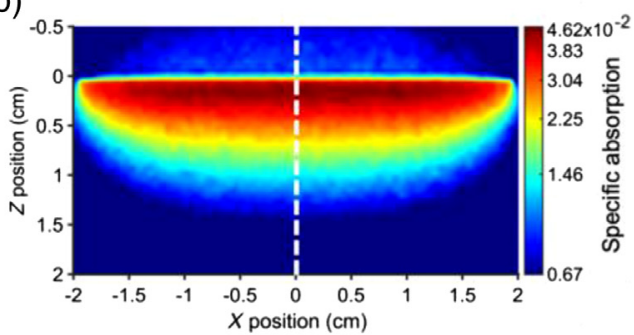

(e)

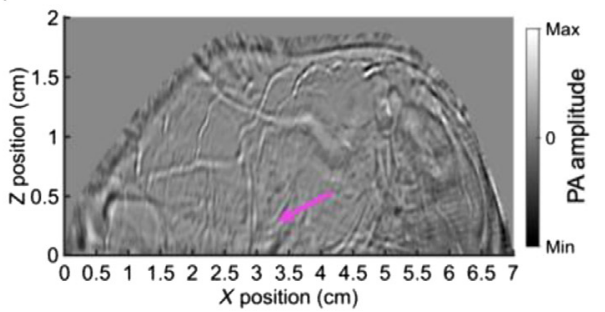

(c)

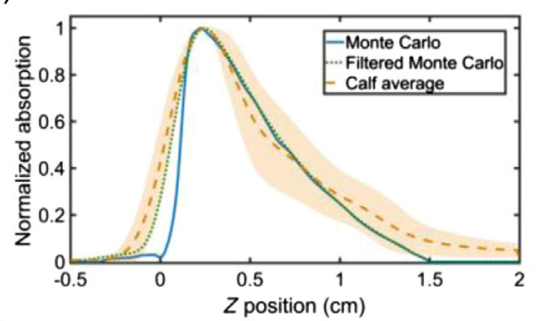

(f)

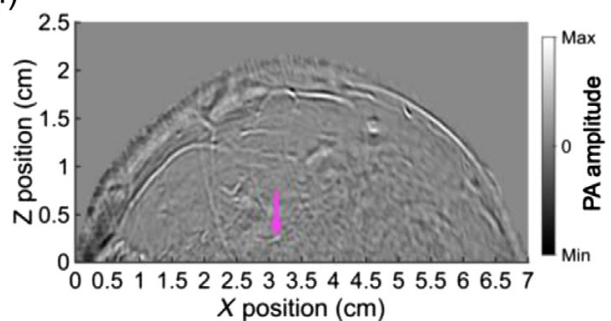

Fig. 4 Analysis of the maximum imaging depth and 1064-nm light absorption in muscular tissue. (a) Cross-sectional image of the volunteer's upper right calf, where vessels and other sharp features were removed using morphological background detection. ${ }^{34}$ (b) Image of the simulated specific absorption (in relative units of $\mathrm{cm}^{-3}$ ) in muscular tissue. In both (a) and (b), the lower bound of the color scale was truncated to the water's average value. (c) Normalized depth-resolved comparison between the simulated-specific absorption in muscular tissue along the white dashed line in (b), before (blue curve) and after (dotted green) applying the background removal filter to the Monte Carlo model, and the experimental average-specific absorption in the volunteer's upper right calf (dashed orange curve) from the four white dotted line profiles in (a). The shaded orange area is the region within one standard deviation of the experimental mean from the four dotted line profiles in (a). The curves were normalized such that the minimum value coming from the water region was set to zero and the maximum value inside the (simulated) tissue was set to one. (d) Fluence compensated unfiltered single-shot cross-sectional image of the volunteer's upper right calf using latitudinal mode illumination. Fluence compensation was performed using the approximate fluence distribution in (a). (e) Magnified region of the volunteer's calf inside the black-dotted rectangle in (d), using the upper right coordinate system. (f) A close-up view of the region outlined by the red-dashed rectangle in (d), using the bottom left coordinate system. 

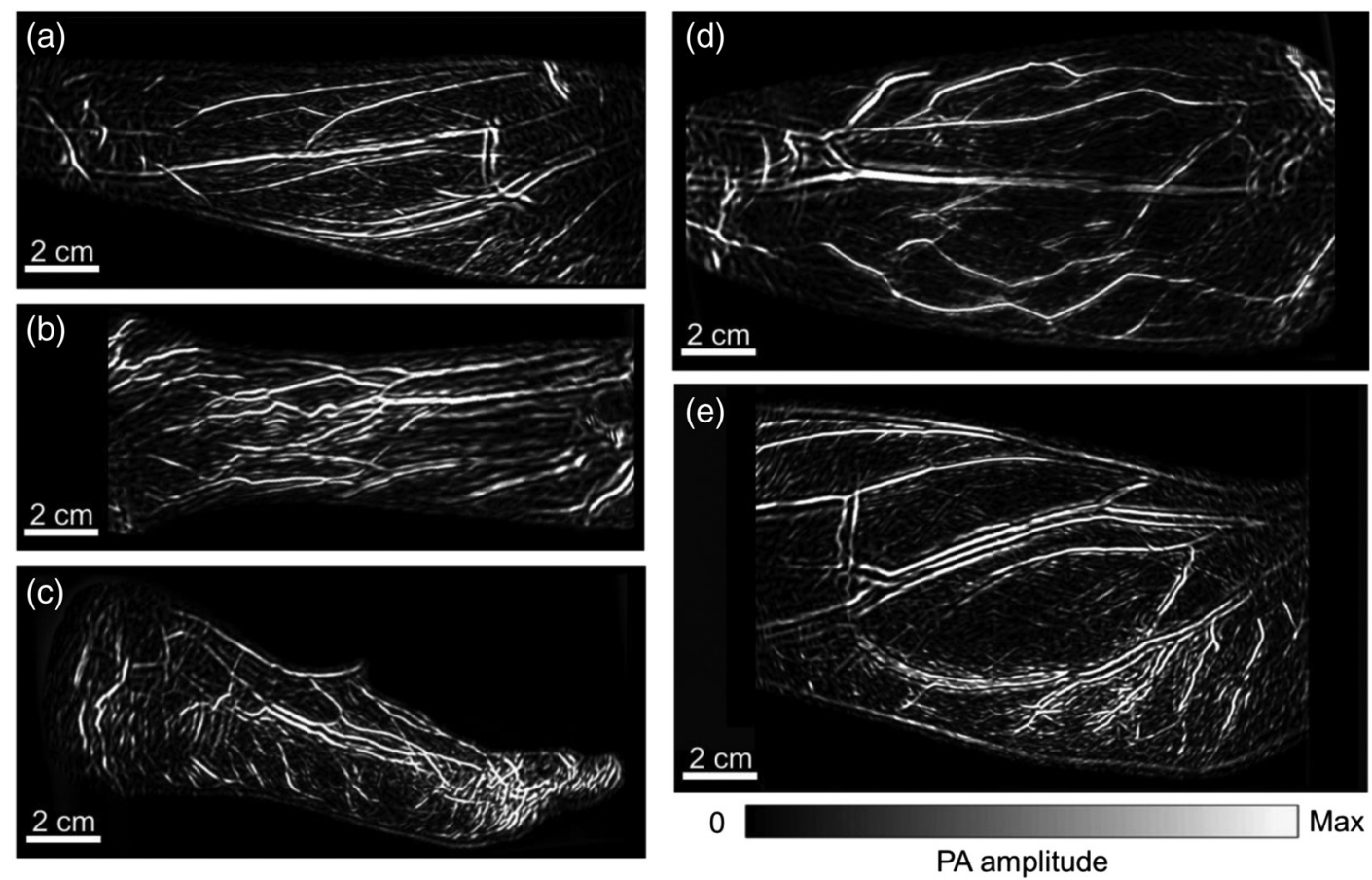

Fig. 5 Photoacoustic images showing the vascular network spanning across different appendages. Latitudinal cross-sectional images of the volunteer's (a) upper right forearm, (b) lower right forearm, ending at the wrist, (c) right foot, (d) right leg, and (e) right bicep.

profile inside the volunteer's calf matched well with the Monte Carlo model [Fig. 4(d)]. At the water to tissue interface, background removal caused a maximum broadening of $\sim 1.4 \mathrm{~mm}$ in the absorption profile of the Monte Carlo model, which was within the standard deviation of the profile seen at the interface of the coupling water and the volunteer's calf. For the Monte Carlo model, the tissue parameters were homogeneous in the $x-y$ plane. The top $0.5-\mathrm{cm}$ layer in the $z$-direction simulated water $\left(\mu_{a}=0.11 \mathrm{~cm}^{-1}\right)^{22,35}$ and the remaining area was muscle tissue $\left(\mu_{a}=0.13 \mathrm{~cm}^{-1}, \mu_{s}=64.60 \mathrm{~cm}^{-1}\right.$, and $\left.g=0.90\right){ }^{22,35}$ Photons $(N=7,245,343)$ started uniformly distributed across the water-tissue surface and propagated within the 3 -D volume $\left(4 \times 4 \times 4 \mathrm{~cm}^{3}\right)$, with a $500-\mu \mathrm{m}$ voxel size. The initial propagation direction of each photon was toward the muscle tissue, at normal incidence (i.e., the focal point is at $z=-\infty$ ). To analyze the maximum achieved imaging depth in muscular tissue, we normalized the single-shot unfiltered PACT image of the volunteer's calf [Fig. 4(d)] by the profile derived in Fig. 4(a). We then magnified the left [Fig. 4(e)] and right [Fig. 4(f)] regions of the volunteer's calf, where each beam from the double-sided illumination entered the tissue at approximately normal incidence. The deepest visible vessel in Fig. 4(e) was at $1.5 \mathrm{~cm}$, with a signal-to-noise ratio (SNR) of 6.1. The deepest visible vessel in Fig. 4(f) was at $1.8 \mathrm{~cm}$, with an SNR of 9.2. The deepest vessels are marked by magenta arrows in Figs. 4(e) and $4(\mathrm{f})$, respectively.

To showcase PACT's ability to image vascular anatomy over a large field of view, in a single laser shot, we used latitudinal mode illumination to obtain planar images of the vascular network spanning across entire appendages. Results for the volunteer's upper and lower forearm, bicep, leg, and foot are shown in Fig. 5. These images show vessels connecting across a distance of $22 \mathrm{~cm}$ (limited by the diameter of the transducer array), clearly visualizing a wide range of vessel sizes.
The smallest detected vessel appears as $255 \mu \mathrm{m}$ in diameter while the largest imaged vessel is $3.8 \mathrm{~mm}$ in diameter (Fig. 6). Vascular diameters were accurately measured by identifying vessel boundaries through a correlation-based template matching method, described in Ref. 23. The capability of endogenously imaging vascular networks across an appendage, in a single-shot, could provide a useful method for physicians to identify vascular abnormalities and understand their effects on the surrounding vascular network.

Finally, we demonstrated that due to the strong vascular contrast and high imaging speed, PACT's resolution is not limited by vascular motion artifacts as the vascular information within the imaging plane can be acquired, with sufficient SNR, in a single laser shot within the acoustic transit time $(\sim 100 \mu \mathrm{s})$. Furthermore, given the sufficiently high laser repetition rate $(10 \mathrm{~Hz})$, we have shown that nonstationary vessels can be monitored, which can be used to derive functional information (Figs. 7 and Video 2). By repeatedly imaging a single plane in the volunteer's wrist, we monitored the vessel wall of a pulsating artery and showed that the pulsation is centered at the heart beat frequency [Fig. 7(b)]. Comparing this motion to a neighboring stationary vessel [Fig. 7(c)], it can be verified that PACT is capable of tracking vessel movement with high contrast. Since vascular obstructions can lead to exaggerated or unusual vessel movement, this high imaging speed could help physicians localize and characterize arterial obstructions. ${ }^{9}$ Furthermore, since arteries pulsate at the heart beat frequency, tracking pulsation could be used to differentiate arteries from veins. ${ }^{23}$

\section{Discussion}

Our work presents a comprehensive study of PACT being used to image the vascular anatomy in human extremities. We can obtain cross-sectional images from a single laser pulse, within $100 \mu \mathrm{s}$, over a 22-cm FOV, with a maximum in-plane resolution of 


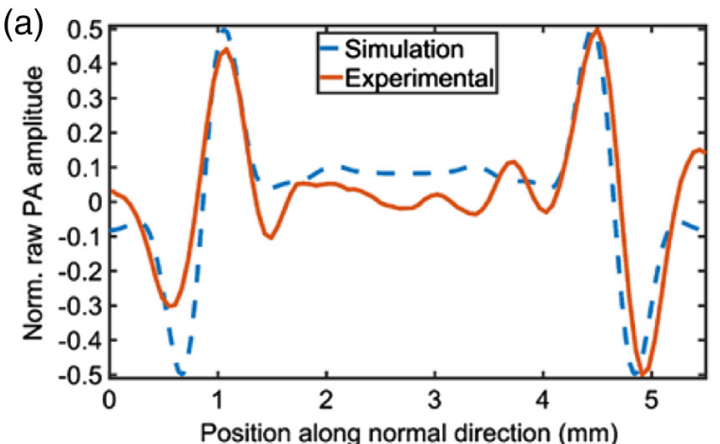

(c)

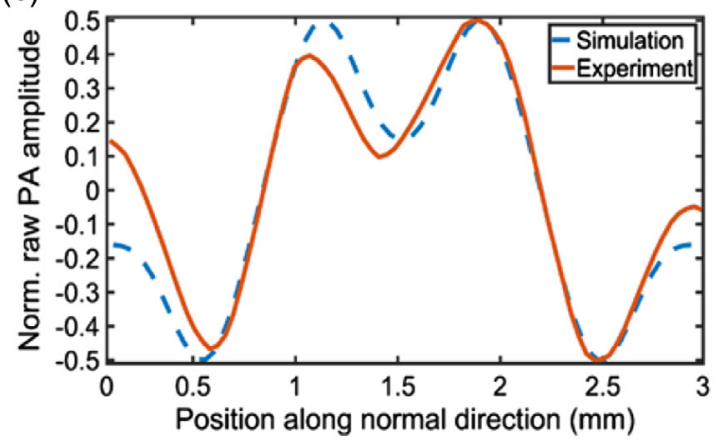

(e)

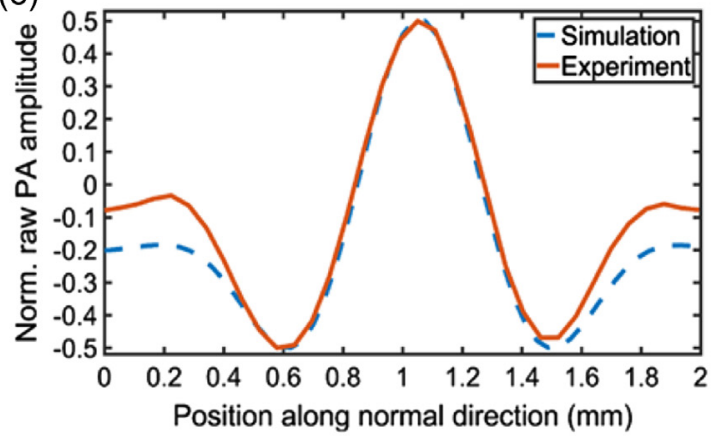

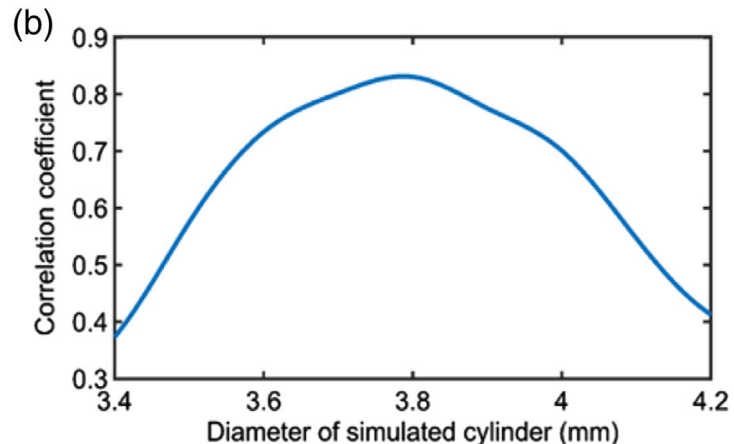

(d)

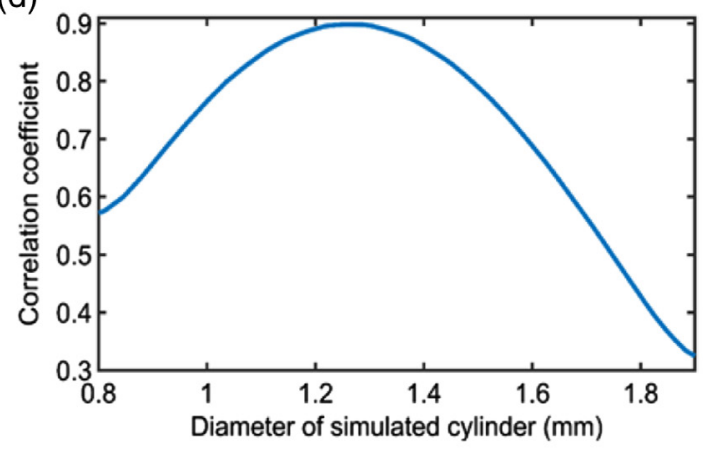

(f)

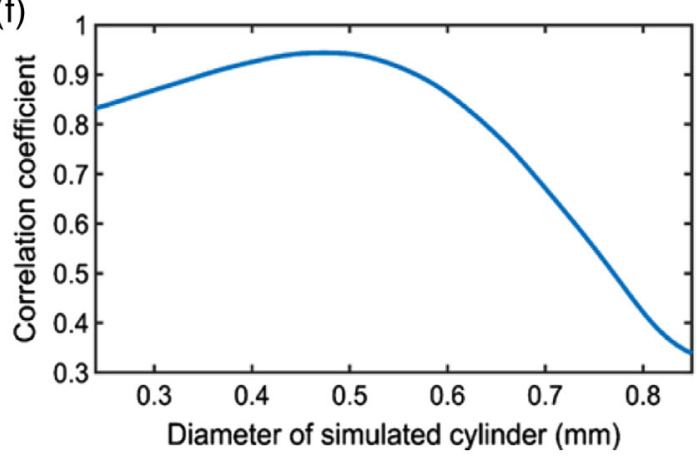

Fig. 6 Quantification of vessel diameter. (a) Normalized raw photoacoustic amplitude across the largest diameter vessel imaged (orange) and across a simulated 3.8- $\mathrm{mm}$ uniform cylinder (dashed blue). (b) Cross-correlation coefficient between the measured vessel in (a) and simulated uniform cylinders near the best match $(3.8 \mathrm{~mm})$. (c) Normalized raw photoacoustic amplitude across a representative medium diameter vessel imaged (orange) and across a simulated 1.26-mm uniform cylinder (dashed blue). (d) Cross-correlation coefficient between the measured vessel in (c) and simulated uniform cylinders near the best match $(1.26 \mathrm{~mm})$. (e) Normalized raw photoacoustic amplitude across a representative small diameter vessel imaged (orange) and across a simulated $0.47 \mathrm{~mm}$ uniform cylinder (dashed blue). (f) Cross-correlation coefficient between the measured vessel in (e) and simulated uniform cylinders near the best match $(0.47 \mathrm{~mm})$. Photoacoustic simulations were done in k-wave. The simulation forward model used a ring array geometry with 1452 transducer elements. The number of elements was chosen such that we were well above the angular Nyquist criteria. ${ }^{27}$ The supported maximum frequency was $20 \mathrm{MHz}$, which was half of the experimental sampling frequency and well past the experimental transducer array's average frequency response $(2.25 \mathrm{MHz}$ center frequency with a $95 \%$ one-way bandwidth). Then, half-time UBP ${ }^{25,26}$ was used on temporally filtered raw channel data. The filterer was a Gaussian fit of the average electrical impulse response from the elements in the experimental transducer array. Filtering the channel data in the time dimension before reconstruction avoided the "inverse crime" as well as provided an accurate simulation of the experimental system's resolution.

$255 \mu \mathrm{m}$. This performance allows us to get snap-shot images of vascular networks in the hands, feet, legs, forearms, and biceps. The ring array geometry provides full-view reconstruction in the imaging plane and complements the general cylindrical structure of each appendage. When scanning in the elevational direction, 3-D images can be obtained over a 7-cm scanning range within $15 \mathrm{~s}$. Since data for a complete cross-sectional area can be collected within $100 \mu \mathrm{s}$ after the laser pulse, we are not subject to heartbeat- or breathing-induced motion artifacts, which distort images in slower imaging modalities. Furthermore, with a laser repetition rate of $10 \mathrm{~Hz}$, we can monitor heartbeat encoded vessel deformations with a $10-\mathrm{Hz}$ frame rate, if no elevational scanning is used. With a maximum imaging depth of $1.8 \mathrm{~cm}$ on either side, we demonstrate complete depth coverage of hands, wrists, and feet for almost all individuals in the United States, based on the national statistics. ${ }^{36}$ We can also cover over $70 \%$ of the soft tissue in the upper forearm, bicep, and leg ${ }^{36}$ while staying within $1 / 16$ to $1 / 8$ the ANSI safety limit. ${ }^{24}$ 
(a)

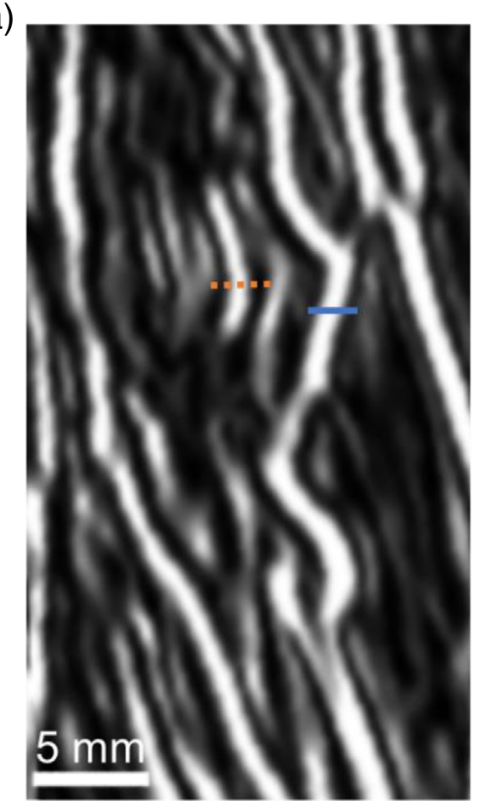

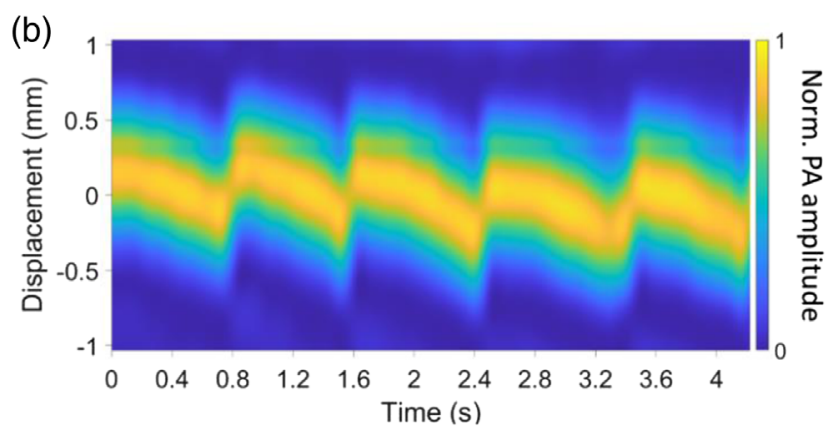

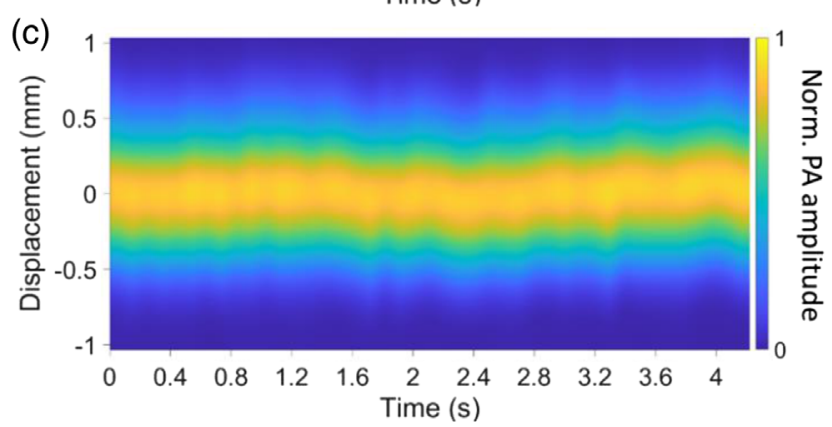

Fig. 7 Functional monitoring of artery deformation due to the heartbeat-induced blood flow (Video 2). (a) Zoomed-in latitudinal cross-sectional PA image of the volunteer's wrist. (b) A 10- $\mathrm{Hz}$ frame rate line profile showing the displacement of the artery wall in (a), marked by the dotted orange line. (c) $\mathrm{A} 10-\mathrm{Hz}$ frame rate line profile showing the displacement of a neighboring vein in (a), marked by the solid blue line (Video 2, MPEG, 2.79 MB [URL: https://doi.org/10.1117/1.JBO.24.2.026003.2]).

The results show that PACT can provide many of the requirements an imaging modality would need to safely image, diagnose, and monitor vascular abnormalities in extremities. The method is noninvasive, provides strong endogenous vascular contrast, and has high spatiotemporal resolution, over a large field of view. Examples of possible applications include screening for peripheral vascular disease and diabetic foot; providing postoperative surveillance for procedures such as revascularization surgery; and monitoring response to drug therapy, such as the perfusion of medication. It is important to note that PACT's penetration depth can be sensitive to skin color since melanosome is the dominant optical absorber in most of the visible and NIR electromagnetic spectrum. The volunteer in this study had a light skin tone, and we would expect volunteers with darker skin to show a more limited penetration depth. Other future research could determine the effectiveness of PACT to diagnose and monitor patients with vascular obstructions or disease. Spectrally resolved PACT could provide multicontrast imaging, such as imaging lipid concentrations in blood vessels for plaque characterization, more accurately pinpointing atherosclerosis, and potentially identifying vulnerable plaques. ${ }^{37-40}$ The ability to perform dynamic studies such as monitoring arterial pulse deformations could provide information for identifying atherosclerosis or other arterial obstructions. Pushing the direction of dynamic studies, following a similar trajectory to the progress in multidetector row CT, multiple acoustic transducer arrays can further reduce scanning time for volumetric imaging.

\section{Disclosures}

Lihong Wang has a financial interest in Microphotoacoustics, Inc., CalPACT, LLC, and Union Photoacoustic Technologies, Ltd., which, however, did not support this work. The remaining authors declare no competing interests.

\section{Acknowledgments}

The authors would like to thank Dr. Junhui Shi and Dr. Konstantin Maslov for their contributions to the system design. This work was sponsored by the United States National Institutes of Health (NIH) grants DP1 EB016986 (NIH Director's Pioneer Award), R01 CA186567 (NIH Director's Transformative Research Award), and R01 NS102213.

\section{References}

1. E. J. Benjamin et al., "Heart disease and stroke statistics-2017 update a report from the American Heart Association," Circulation 135(10), E146-E603 (2017).

2. C. Y. Ho and C. M. Shanahan, "Medial arterial calcification: an overlooked player in peripheral arterial disease," Arterioscler. Thromb. Vasc. Biol. Rev. 36(8), 1475-1482 (2016).

3. L. C. de Vos et al., "Skin autofluorescence is associated with 5-year mortality and cardiovascular events in patients with peripheral artery disease," Arterioscler. Thromb. Vasc. Biol. 34(4), 933-938 (2014).

4. A. T. Hirsch et al., "Peripheral arterial disease detection, awareness, and treatment in primary care," JAMA-J. Am. Med. Assoc. 286(11), 1317-1324 (2001).

5. J. M. Tarkin et al., "Imaging atherosclerosis," Circ. Res. 118(4), 750-769 (2016).

6. A. Christie and G. Roditi, "Vascular imaging: the evolving role of the multidisciplinary team meeting in peripheral vascular disease," Semin. Interventional Radiol. 31(4), 320-329 (2014).

7. A. Micari et al., "New imaging modalities in peripheral interventions," Eur. Heart J. Suppl. 17(A), A18-A22 (2015).

8. A. W. Pollak, P. T. Norton, and C. M. Kramer, "Multimodality imaging of lower extremity peripheral arterial disease current role and future directions," Circ. Cardiovasc. Imaging 5(6), 797-807 (2012).

9. M. A. Khalil et al., "Detection of peripheral arterial disease within the foot using vascular optical tomographic imaging: a clinical pilot study," Eur. J. Vasc. Endovasc. Surg. 49(1), 83-89 (2015).

10. L. Norgren et al., "Inter-society consensus for the management of peripheral arterial disease (TASC II)," J. Vasc. Surg. 45, S5-S67 (2007). 
11. M. C. J. M. Kock et al., "Multi-detector row computed tomography angiography of peripheral arterial disease," Eur. Radiol. 17(12), 3208-3222 (2007).

12. K. J. Murphy, J. A. Brunberg, and R. H. Cohan, "Adverse reactions to gadolinium contrast media: a review of 36 cases," Am. J. Roentgenol. 167(4), 847-849 (1996)

13. M. A. Perazella, "Current status of gadolinium toxicity in patients with kidney disease," Clin. J. Am. Soc. Nephrol. 4(2), 461-469 (2009).

14. R. J. Hooley, L. M. Scoutt, and L. E. Philpotts, "Breast ultrasonography: state of the art," Radiology 268(3), 642-659 (2013).

15. A. C. S. Tan et al., "An overview of the clinical applications of optical coherence tomography angiography," Eye 32(2), 262-286 (2017).

16. J. J. Xu et al., "Long ranging swept-source optical coherence tomography-based angiography outperforms its spectral-domain counterpart in imaging human skin microcirculations," J. Biomed. Opt. 22(11), 116007 (2017).

17. A. Deegan et al., "Optical coherence tomography angiography of skin with healthy and inflammatory dermatologic conditions," Lasers Surg. Med. 50(3), 183-193 (2017).

18. D. Razansky et al., "Multispectral opto-acoustic tomography of deepseated fluorescent proteins in vivo," Nat. Photonics 3(7), 412-417 (2009).

19. A. Taruttis and V. Ntziachristos, "Advances in real-time multispectral optoacoustic imaging and its applications," Nat. Photonics 9(4), 219-227 (2015)

20. J. J. Yao, J. Xia, and L. V. Wang, "Multiscale functional and molecular photoacoustic tomography," Ultrason. Imaging 38(1), 44-62 (2016).

21. Y. Zhou, J. J. Yao, and L. H. V. Wang, "Tutorial on photoacoustic tomography," J. Biomed. Opt. 21(6), 061007 (2016).

22. S. L. Jacques, "Optical properties of biological tissues: a review," Phys. Med. Biol. 58(11), R37-R61 (2013)

23. L. Lin et al., "Single-breath-hold photoacoustic computed tomography of the breast," Nat. Commun. 9, 2352 (2018).

24. American National Standards Institute, "American national standard for the safe use of lasers ANSI z 1/36.1-2007," Laser Institute of America, Orlando, Florida, https://webstore.ansi.org/Standards/LIA/ ANSIZ1362007 (2007).

25. M. A. Anastasio et al., "Half-time image reconstruction in thermoacoustic tomography," IEEE Trans. Med. Imaging 24(2), 199-210 (2005).

26. M. H. Xu and L. H. V. Wang, "Universal back-projection algorithm for photoacoustic computed tomography," Phys. Rev. E 71(1), 016706 (2005).

27. Y. Xu, M. H. Xu, and L. H. V. Wang, "Exact frequency-domain reconstruction for thermoacoustic tomography-II: cylindrical geometry," IEEE Trans. Med. Imaging 21(7), 829-833 (2002).

28. L. Li et al., "Single-impulse panoramic photoacoustic computed tomography of small-animal whole-body dynamics at high spatiotemporal resolution," Nat. Biomed. Eng. 1, 0071 (2017).

29. P. P. Bruyant, J. Sau, and J.-J. Mallet, "Streak artifact reduction in filtered backprojection using a level line-based interpolation method," J. Nucl. Med. 41(11), 1913-1919 (2000).

30. L. I. Rudin, S. Osher, and E. Fatemi, "Nonlinear total variation based noise removal algorithms," Phys. D 60(1-4), 259-268 (1992).

31. F. Shi and J. Yang, "Multiscale vesselness based bilateral filter for blood vessel enhancement," Electron. Lett. 45(23), 1152-1153 (2009).
32. P. F. Hemler, E. S. McCreedy, and M. J. McAuliffe, "Performance evaluation of multiscale vessel enhancement filtering," Proc. SPIE 5370, 1785-1794 (2004).

33. A. F. Frangi et al., "Multiscale vessel enhancement filtering," Lect. Notes Comput. Sci. 1496, 130-137 (1998).

34. P. Hai et al., "Near-infrared optical-resolution photoacoustic microscopy," Opt. Lett. 39(17), 5192-5195 (2014)

35. G. Wang et al., "Morphological background detection and illumination normalization of text image with poor lighting," PLoS One 9(11), e110991 (2014).

36. C. Gordon et al., "Anthropometric Survey of U.S. Army Personnel: summary statistics," Interim Report for 1988, https://apps.dtic.mil/ docs/citations/ADA209600 (1989).

37. L. Lin et al., "In vivo photoacoustic tomography of myoglobin oxygen saturation," J. Biomed. Opt. 21(6), 061002 (2016).

38. C. Casavola et al., "Blood flow and oxygen consumption with nearinfrared spectroscopy and venous occlusion: spatial maps and the effect of time and pressure of inflation," J. Biomed. Opt. 5(3), 269-276 (2000).

39. M. Wu et al., "Real-time volumetric lipid imaging in vivo by intravascular photoacoustics at 20 frames per second," Biomed. Opt. Express 8(2), 943-953 (2017).

40. P. Wang et al., "High-speed intravascular photoacoustic imaging of lipid-laden atherosclerotic plaque enabled by a $2-\mathrm{kHz}$ barium nitrite Raman laser," Sci. Rep. 4, 6889 (2014).

Parker Wray is a student at California Institute of Technology, Pasadena, California, USA. He received his BS degree in electrical engineering from the University of Texas at Austin, Austin, Texas, USA, in 2015, and his MS degree in electrical engineering from California Institute of Technology in 2018. His current research interests include photoacoustic computed tomography for small animal and human imaging as well as photoacoustic microscopy for subcellular imaging.

Li Lin is currently a PhD candidate at California Institute of Technology, under the tutelage of Professor Lihong V. Wang. He earned his master's degree at the University of Pennsylvania in 2013 and his bachelor's degree at Tianjin University in 2011. His research focuses on photoacoustic computed tomography and microscopy, specifically in human breast and animal brain imaging.

Peng $\mathrm{Hu}$ is a $\mathrm{PhD}$ candidate at the Washington University in St. Louis. $\mathrm{He}$ received his BS and MS degrees from Wuhan University, Wuhan, China, in 2012 and 2015, respectively. His research interests include numerical computation, image reconstruction and processing for photoacoustic tomography, compressed sensing, and deep learning.

Lihong V. Wang received his PhD from Rice University, Houston, Texas, USA. Currently, he is holding the Bren Professor of Medical Engineering and Electrical Engineering at California Institute of Technology. He has published 495 peer-reviewed journal articles and delivered 500 keynote, plenary, or invited talks. His Google scholar h-index and citations have reached 124 and over 64,000, respectively. He was inducted into the National Academy of Engineering. 